\title{
CORRIGENDUM
}

\section{Corrigendum: Efficacy and safety of photodynamic therapy with RLP068 for diabetic foot ulcers: a review of the literature and clinical experience}

\section{Abstract}

The authors wish to make the following corrections to their article:
Pantò F, Adamo L, Giordano C, Licciardello C. Efficacy and safety of photodynamic therapy with RLP068 for diabetic foot ulcers: a review of the literature and clinical experience. Drugs in Context 2020; 9: 2019-10-3. DOI: 10.7573/dic.2019-10-3

\section{Corrigendum}

The authors regret that there are errors in their original paper.

1. Legends to Figures 3 and 4 . The following shows where original text should be removed (strikethrough) and new wording introduced (underlined).

Figure 3. Antimicrobial PDT with RLP068, in addition to ceftriaxone. (A) Patient condition before treatments. Patient condition after 8 (B) and $16(C)$ applications of RLP068-PDT (B) and (C) at 10 days from the beginning of treatment.

Figure 4. Antimicrobial PDT with RLP068, in addition to amoxicillin/clavulanic acid. (A) Patient condition before treatments. Patient condition after $4(B)$ and $16(C)$ applications of RLP068-PDT (B) and (C) at 1 month from the beginning of treatment. 